Research Paper

\title{
Cestode Antigens Induce a Tolerogenic-Like Phenotype and Inhibit LPS In- flammatory Responses in Human Dendritic Cells
}

\section{César A. Terrazas ${ }^{3^{*}}$, Fausto Sánchez-Muñoz ${ }^{1^{*}}$, Ana M. Mejía-Domínguez², Luis M. Amezcua-Guerra1, Luis I. Terrazas $^{3}$, Rafael Bojalil ${ }^{1,4}$, Lorena Gómez-García ${ }^{1 凶}$}

1. Department of Immunology, Instituto Nacional de Cardiología Ignacio Chávez, México City, México

2. Blood Bank, Instituto Nacional de Cardiología Ignacio Chávez, México City, México

3. Biomedicine Unit, Facultad de Estudios Superiores Iztacala, UNAM, México City, México

4. Department of Health Care, Universidad Autónoma Metropolitana Xochimilco, México City, México

* These two authors contributed equally to this work.

$\triangle$ Corresponding author: lorena.gomez@cardiologia.org.mx

(C) Ivyspring International Publisher. This is an open-access article distributed under the terms of the Creative Commons License (http://creativecommons.org/ licenses/by-nc-nd/3.0/). Reproduction is permitted for personal, noncommercial use, provided that the article is in whole, unmodified, and properly cited.

Received: 2011.09.01; Accepted: 2011.10.01; Published: 2011.11.01

\begin{abstract}
Pathogens have developed strategies to modify Dendritic Cells (DCs) phenotypes and impair their functions in order to create a safer environment for their survival. DCs responses to helminths and their derivatives vary among different studies. Here we show that excretory/secretory products of the cestode Taenia crassiceps (TcES) do not induce the maturation of human DCs judged by a lack of increment in the expression of CD83, HLA-DR, CD80 and CD86 molecules but enhanced the production of IL-I0 and positively modulated the expression of the C-type lectin receptor $M G L$ and negatively modulated the expression of DC-SIGN. Additionally, these antigens were capable of down-modulating the inflammatory response induced by LPS in these cells by reducing the expression of the maturation markers and the production of the inflammatory cytokines IL-I $\beta$, TNF, IL-I2 and IL- 6 . The effects of TcES upon the DCs responses to LPS were stronger if cells were exposed during their differentiation to the helminth antigens. All together, these findings suggest the ability of TcES to induce the differentiation of human DCs into a tolerogenic-like phenotype and to inhibit the effects of inflammatory stimuli.
\end{abstract}

Key words: Dendritic cells, tolerogenic phenotype, Taenia crassiceps.

\section{Introduction}

Dendritic cells fulfill a major role in the immune system directing the kind and the intensity of the response mounted against the diverse antigens and autoantigens encountered [1]. Thus, these cells can determine whether immunity or tolerance is exerted among the plethora of possible stimuli [2]. Different DCs subsets [3-4], phenotypes [5-6] and even microenvironments to which these cells are exposed [7-8], may determine the type of immune response induced by them. However, in general terms, immature DCs (iDCs) that face a pathogenic stimulus will enter in a process characterized by the expression of maturation and co-stimulation molecules as well as the production of cytokines that promote and modulate inflammation and effector cell functions, including IL-12, IL-1 $\beta$, IL-6, TNF and IL-8 [1]. All these features allow DCs to trigger full lymphocyte responses [1]. On the contrary in a steady state, DCs will not display an enhanced expression of maturation markers and they will rather show an increased secretion of regulatory cytokines such as IL-10 and TGF- $\beta$ [9-11]. Interestingly, these DCs have been described as cells capable 
of inducing tolerance instead of immunoreactivity [12]. DCs express a wide repertory of receptors that allow them to interact with pathogens, tissue-derived danger signals and self-antigens [1]. Among these, Toll-like receptors (TLRs) and C-type lectin receptors (CLRs) are differentially expressed by subsets [13] and perhaps phenotypes [14] of DCs. The cross-talk known to occur between these receptors can either inhibit or enhance specific signals in DCs, thereby modulating their phenotype and function and accounting for the different pathways to tolerance or immune activation [13, 15-17]. Since changes in the expression or activity of CLRs and TLRs can influence crucial aspects of the immune response, targeting these receptors is not surprisingly one of the mechanisms that parasites presumably use to impair DCs function and improve their survival within the host [18-19].

Helminth antigens have been a matter of intense research during the last years due to their capacity to modulate immune responses [20]. Described modulatory properties range from suppression of antigen-specific and unspecific $T$ cell responses [21-22] to induction of specific cells responses such as alternatively activated macrophages and regulatory $\mathrm{T}$ cells [23-24]. In recent years, it has been shown that helminth antigens can also affect the response of DCs, presumably as a strategy to divert the immune response elicited against them [25]. Nevertheless it has been demonstrated that different helminth-derived molecules can affect DCs responses, it has not been possible to define a general phenotype induced by these parasites since distinct effects upon these cells have been observed. For instance, while some studies have shown that helminth antigens derived from Ascaris suum [26], Schistosoma mansoni [27], Nippostrongylus brasiliensis [28] or Fasciola hepatica [29] induce costimulatory molecule expression on DCs and production of inflammatory cytokines as IL-12p40, TNF or IL-6, other studies using Heligmosomoides polygyrus [24] and once again S. mansoni [30], have reported immature phenotypes with no cytokine secretion, indicating that different helminths and even different molecules derived from the same helminth can affect DCs activity in various ways.

To date most studies have addressed the activity that nematode and trematode-derived antigens exert upon DCs while the effects of cestode antigens on these cells are much less well explored. Most importantly, most of these studies have been done using mouse-derived DCs limiting our knowledge regarding the modulatory activity of helminth-derived antigens on human DCs. Taenia crassiceps is a cestode parasite of rodents and canines that shares a remark- able antigenic resemblance with the cestode parasite of humans Taenia solium [31-32]; thus, this parasite has been used as a model for the study of cysticercosis for many years [33-34]. Recently, we described that Taenia crassiceps excreted-secreted antigens (TcES) impair murine bone marrow-derived DCs (BMDCs) function down-modulating their TLR-mediated pro-inflammatory activity and inducing Th2 responses in allogenic assays [35]. However, the activity of these modulatory antigens on human DCs has not been addressed to date.

Here we explored the capacity of TcES to modulate the phenotype of human DCs responses. We found that despite the source of these antigens is not a human parasite, they can indeed modify human DCs phenotype and function. TcES did not induce maturation of DCs but instead down-modulated the expression of maturation and co-stimulatory molecules in response to the pro-inflammatory stimulus LPS. Even more, TcES enhanced production of IL-10 by these DCs but not IL-12, IL-1 $\beta$, TNF and IL-6. Also, TcES dramatically reduced the production of these pro-inflammatory cytokines after LPS stimulation and this effect was more pronounced when DCs were exposed to the antigens when they were being differentiated from monocytes. Moreover, TcES modulated the expression of PRRs involved on key functions of DCs such as DC-SIGN and MGL. All together, these results indicate that TcES induce a tolerogenic-like phenotype in human DCs and down-modulate the inflammatory response that LPS induces in these cells.

\section{Materials and Methods}

\section{Parasites and antigens}

Metacestodes of $T$. crassiceps were harvested under sterile conditions from the peritoneal cavity of female Balb/c mice after 2-4 months of infection. The cysticerci were washed four times with sterile PBS and maintained in culture in PBS at $37^{\circ} \mathrm{C}$ for $24 \mathrm{~h}$. TcES were recovered from the supernatant and centrifuged for $10 \mathrm{~min}$ at $1000 \mathrm{~g}$. The upper fraction was concentrated using 50kDa Amicon Ultra Filter (Millipore). Protease inhibitors were added to the $\geq 50 \mathrm{kDa}$ fraction and samples were stored at $-70^{\circ} \mathrm{C}$ until further use.

\section{Monocyte-derived dendritic cells}

Human peripheral blood mononuclear cells (PBMC) were obtained from buffy coats of 15 healthy blood donors from the Instituto Nacional de Cardiología Ignacio Chávez's blood bank. Informed consent was obtained for the use of blood samples according to the declaration of Helsinki and the local scientific and ethics committees approved the proto- 
col. PBMC were isolated by Ficoll-gradient centrifugation (GE Healthcare), analyzed in a Coulter AcT for cellular types (Beckman Coulter) and $3 \times 10^{6}$ monocytes were left to adhere in 6-well culture plates for $2 \mathrm{~h}$. After this period, non-adherent cells were washed away and adherent cells were cultured in RPMI medium supplemented with $10 \%$ SFB and penicillin/streptomycin in presence of $400 \mathrm{U} / \mathrm{ml}$ of IL-4 and $800 \mathrm{U} / \mathrm{ml}$ of GM-CSF during 6 days with replacement of medium and cytokines at day 3 . For experiments assessing the effects of TcES during DC differentiation cells received $20 \mathrm{ug} / \mathrm{ml}$ of TcES at day 0 and 3 (pre-stimulation). At day 6 non-adherent cells were recovered and placed for $24 \mathrm{~h}$ in fresh medium. At this point we determined by flow cytometry the percentage of $\mathrm{CD} 11 \mathrm{c}+$ cells and for all experiments this was $\geq$ $80 \%$. At day 7, both pre-stimulated and not pre-stimulated cells were challenged with $20 \mathrm{ug} / \mathrm{ml}$ TcES, $1 \mathrm{ug} / \mathrm{ml}$ LPS or a combination of them for $24 \mathrm{~h}$. For the real-time PCR analysis cells were stimulated for a period of $3 \mathrm{~h}$ using the same stimuli. DCs derived from donors numbered 1-6 were used for the analysis of cell surface markers. DCs derived from donors numbered 1-9 were used for the analysis of cytokine production by ELISA. DCs derived from donors 10-15 were used for the analysis of mRNA expression. $1 \mathrm{X}$ $10^{6}$ DCs were used for each assay.

\section{Quantification of cytokine production}

Supernatants were recovered after the $24 \mathrm{~h}$ stimulation period and production of the cytokines TNF, IL-6, IL-12, IL-10 (Peprotech), IL-23, IL-1 $\beta$ and TGF- $\beta$ (R\&D) was measured by ELISA kits in the supernatants of DCs cultures. For the analysis of cytokine mRNAs levels cells were recovered from cultures $3 \mathrm{~h}$ post-stimulation.

Table I - Primers for RT-qPCR.

\begin{tabular}{|c|c|c|c|c|}
\hline Gene & GENEBANK & PRIMERS (5'-3’) & $\begin{array}{l}\text { Amplicon } \\
\text { Size (bp) }\end{array}$ & $\begin{array}{l}\text { PROBE } \\
\text { UPL }\end{array}$ \\
\hline \multirow[t]{2}{*}{ TLR2 } & NM_003264.3 & CGTTCTCTCAGGTGACTGCTC & 66 & $\# 14$ \\
\hline & & TCTCCTTTGGATCCTGCTTG & & \\
\hline \multirow[t]{2}{*}{ TLR4 } & NM_138554.2 & CTGCGTGAGACCAGAAAGC & 75 & \#33 \\
\hline & & TTCAGCTCCATGCATTGATAA & & \\
\hline \multirow[t]{2}{*}{ CD209 (DC SIGN) } & NM_021155.3 & CCCAGCTCGTCGTAATCAA & 80 & \#73 \\
\hline & $\begin{array}{l}\text { NM_001144893.1 } \\
\text { NM_001144894.1 } \\
\text { NM_001144895.1 } \\
\text { NM_001144896.1 } \\
\text { NM_001144897.1 } \\
\text { NM_001144899.1 }\end{array}$ & CCAGGTGAAGCGGTTACTTC & & \\
\hline \multirow[t]{2}{*}{ M6PR (Manose Receptor) } & NM_002355.2 & GCTGGAGGACTGGACTGCTA & 62 & \#76 \\
\hline & & TGTCTGCCAGGATTCTCTCAC & & \\
\hline CLEC10A (Clec) & NM_006344.2 & GAATCACACССТCCAGACCTC & 83 & \#16 \\
\hline
\end{tabular}

\section{Analysis of cell surface markers}

Surface expression of maturation and co-stimulatory molecules was analyzed using multicolor flow cytometry. Human DCs were harvested post-stimulation, washed and suspended in FACS buffer (PBS/FBS 5\%/0.05\% $\mathrm{NaN}_{3}$ ). Fc receptors were blocked with $1 \mu \mathrm{g} / \mu \mathrm{l}$ of human IgG for $30 \mathrm{~min}$ at $4^{\circ} \mathrm{C}$. Cells were washed and triple stained with an APC-conjugated antibody against CD11c, FITC conjugated antibodies against Human Leukocyte Antigen-DR (HLA-DR) and CD80 and PE-conjugated antibodies against CD83 and CD86 (all antibodies from Biolegend). Cells were analyzed on a FACsCalibur flow cytometer using Cell Quest software (Beckton Dickinson).

\section{Quantitative real-time PCR}

Cells $\left(10^{6}\right)$ were lysed in $1000 \mu \mathrm{l}$ TRIzol (Invitrogen) and incubated for $5 \mathrm{~min}$ at RT in presence of $1 \mathrm{ul}$ of Glycogen (20ug/ul stock) (Invitrogen). RNA was subsequently isolated from the water phase according to the manufacturer's instruction. The cDNA was generated using $250 \mathrm{ng}$ of total RNA, random hexamers and the Transcriptor first strand cDNA synthesis kit (ROCHE). RT-qPCR analysis was performed using the LightCycler 2.0 system (ROCHE) with LNA hydrolysis probes from the Universal Probe Library Roche (UPL), and intron spanning primers (Table 1) from Invitrogen. One $\mu \mathrm{l}$ of cDNA was amplified with $400 \mathrm{nM}$ of primers, $100 \mathrm{nM}$ of UPL probe, with the LighCycler TaqMan ${ }^{\circledR}$ Master (ROCHE) followed by 45 cycles of $95^{\circ} 10 \mathrm{sec}$. $60^{\circ} 30 \mathrm{sec}$., and $72^{\circ} 1 \mathrm{sec}$. Reference genes $\mathrm{ACTB}$ and GAPDH transcripts were used for relative quantification. The mRNA relative quantification of target genes was conducted using the LightCycler software 4.1 , according to the $2-\Delta \Delta \mathrm{Ct}$ method. The calibrator sample employed was the non-stimulated DCs. 


\begin{tabular}{|c|c|c|c|c|}
\hline & NM_182906.2 & TCTGAGGTTGTCACAGCTGAA & & \\
\hline \multirow[t]{2}{*}{ IL23 } & NM_016584.2 & TGTTCCCCATATCCAGTGTG & 77 & \#76 \\
\hline & & TCCTTTGCAAGCAGAACTGA & & \\
\hline \multirow[t]{2}{*}{ TGFB1 } & NM_000660.3 & GCAGCACGTGGAGCTGTA & 76 & \#72 \\
\hline & & CAGCCGGTTGCTGAGGTA & & \\
\hline \multirow[t]{2}{*}{$A C T B$} & NM_001101.3 & CCAACCGCGAGAAGATGA & 97 & \#64 \\
\hline & & CCAGAGGCGTACAGGGATAG & & \\
\hline \multirow[t]{2}{*}{ GADPH } & NM_002046.3 & AGCCACATCGCTCAGACAC & 66 & $\# 60$ \\
\hline & & GCCCAATACGACCAAATCC & & \\
\hline
\end{tabular}

RT-qPCR assays were intron spaning. DC SIGN and CLEC isoforms mRNAs enlisted were detected by the assays.

\section{Statistics}

Data are expressed as mean and standard errors. Comparisons were performed by the Wilcoxon signed rank (matched pairs) test. Significance was set on a $p$ value $<0.05$. All analyses were performed with the GraphPad Prism v. 5 statistical software.

\section{Results}

\section{Human DCs exposed to TcES show an imma- ture-like phenotype}

The maturation status of DCs can shape the balance to tolerance or immunity. Interestingly, different pathogens can interfere with this process on DCs hence affecting the infection outcome $[2,25]$.To determine the maturation status of human DCs exposed to TcES, LPS or both, we performed three-color cytometric analysis using the molecules HLA-DR (MHCII), CD83, CD80 and CD86 as markers.

We found that TcES, the excreted-secreted antigens of the cestode $T$. crassiceps, did not induce maturation of human DCs as judged by the absence of up-regulation (compared to those that only received medium) of any of the maturation and co-stimulation molecules tested in this study (Fig.1A). As expected, DCs that received LPS expressed HLA-DR molecules and showed a significant increment in CD83, CD80 and CD86 expression compared to non-stimulated cells, indicating they were fully mature (Fig 1A). However, when these cells were exposed to a combination of LPS plus TcES, the presence of CD83, CD80 and CD86 dropped, suggesting that TcES interfere with the typical maturation process induced by pro-inflammatory stimuli such as LPS.

Others have reported that monocytes exposed to helminth antigens have an impaired capability to differentiate into DCs [36-37]. In addition, infection with metacestodes is accompanied by antigens in circulation [38-39] that might affect the activity of monocytes. To assess if the differentiation of monocytes into DCs was affected by TcES we added these antigens to our cultures at day 0 and 3 . We found that there was no difference in the proportion of $\mathrm{CD} 11 \mathrm{c}+$ cells between cultures that received TcES during their differentiation and the ones that received it only as a final challenge, indicating that TcES did not affect the capability of human monocytes to differentiate into DCs (Fig 1B). Additionally, DCs that received TcES during their differentiation and were re-challenged at day 7 with TcES, LPS or the combination of them, responded in the same way as the cells that received the antigens only when they were mature; this is, those exposed to TcES showed an immature phenotype and the ones exposed to LPS plus TcES expressed significantly less maturation and co-stimulation molecules compared to those that received only LPS. An exception was found for CD83, where early exposure to TcES affected significantly its expression after LPS stimulation when compared to DCs that were not exposed to such antigens during differentiation (Fig 1B).

\section{TcES induce production of IL- I 0 by human DCs and impaired their LPS-inflammatory cytokine response}

It is well known that soluble factors released by DCs after pathogen exposure are considered the third signal needed for lymphocyte activation and polarization of immune responses [40]. In contrast to those related to exposure to virus, bacteria or fungi, the soluble factors released by DCs after exposure to helminths are less well characterized. Human DCs phenotype in response to TcES, LPS and LPS/TcES was also assessed by determining their cytokine profile.

In our experiments, TcES failed to induce production of the cytokines IL-12, TNF, IL-1 $\beta$ and IL- 6 by human DCs but interestingly these antigens induced the production of the regulatory cytokine IL-10. When DCs were stimulated with LPS, levels of all the cytokines were enhanced (Fig. 2) however; combination of LPS and TcES diminished cytokine LPS response of IL-12, TNF, IL-1 $\beta$ and IL-6, leaving IL-10 production unchanged. 
The levels of IL-23 and TGF- $\beta$ detected by ELISA were too low to make a proper quantification of them, so we decided to determine the transcription of mRNA of these cytokines by RT-qPCR. We found that as expected, LPS enhanced the transcripts of the inflammatory cytokine IL-23 and the addition of TcES did not influence this increment. TcES by itself did not induce an up-regulation of this messenger confirming its non pro-inflammatory activity (Fig. 2B). Even when the expression of TGF- $\beta$ was somewhat higher in response to TcES, it was not statistically significant (Fig. 2B)

Interestingly, when human DCs were exposed to TcES during their differentiation, the effect of these antigens on LPS-induced cytokine production was more pronounced. In this scenario, LPS-induced production of IL-12, TNF and IL-6 was significantly down-regulated in cells that received TcES during their differentiation compared to those cells that did not. The addition of TcES plus LPS reduced even further the production of IL-12 and TNF (Fig. 3).
A)
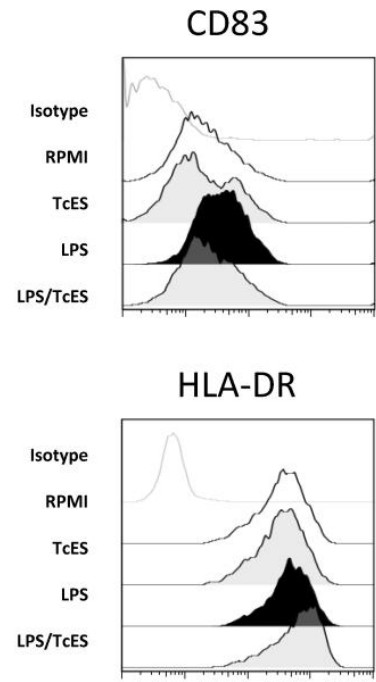

CD80

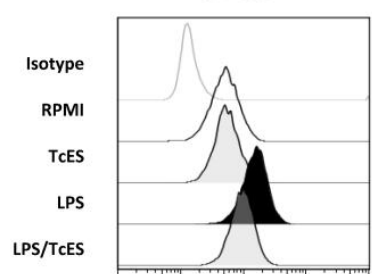

CD86

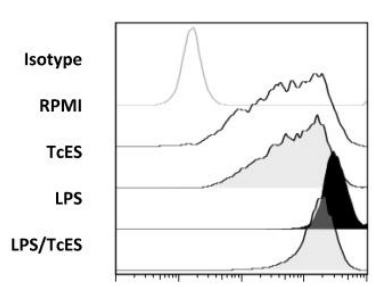

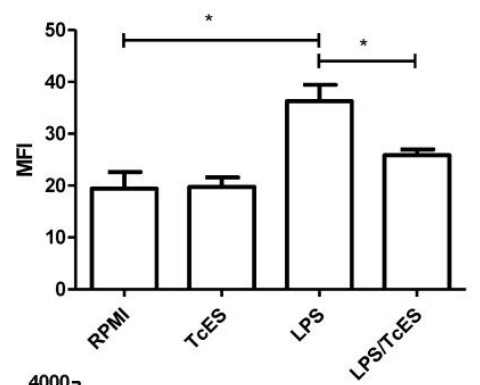

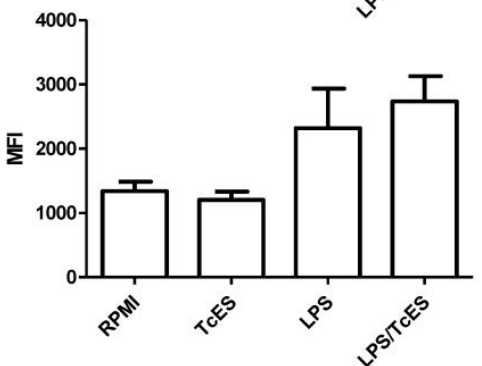

B)

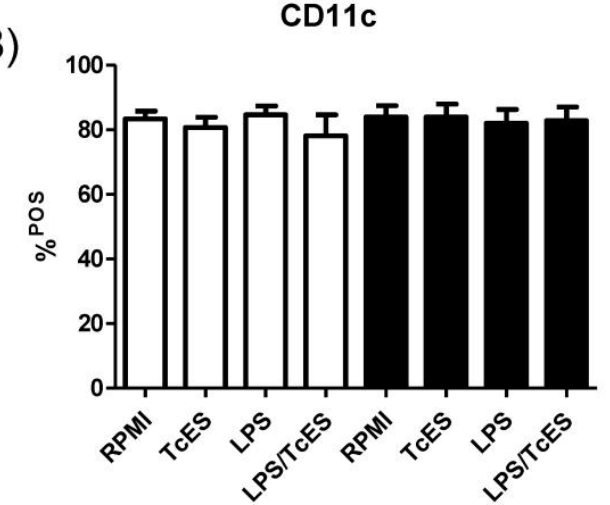

CD83

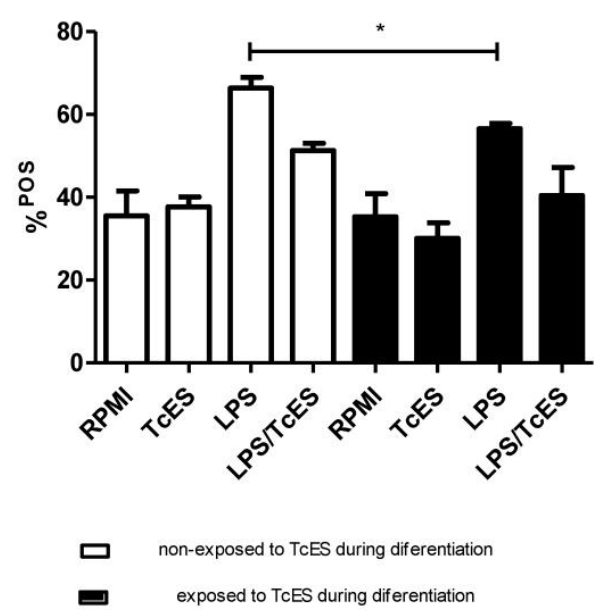

Figure I. Expression of maturation markers in human DCs induced by TcES. A) iDCs were stimulated with $20 \mu g$ of TcES, I $\mu \mathrm{g}$ of LPS or a combination of them and 24h post-stimulation were stained with anti-CD83, anti-CD80, anti-CD86 and anti-HLA-DR and analyzed by flow-cytometry. B) iDCs stimulated with $20 \mu \mathrm{g}$ of TcES, I $\mu \mathrm{g}$ of LPS or a combination of them vs human monocytes that were exposed to $20 \mathrm{ug} / \mathrm{ml}$ of TcES at day 0 and 3 of their differentiation to DCs. Once differentiated in iDCs they were stimulated with LPS, LPS+TcES or TcES alone. DCs derived from donors I-6 (six donors) were used for this analysis. Assays were performed once for each donor. Data represents means \pm standard error of six independent experiments. $* \mathrm{P}<0.05$ was deemed significant. 
A)

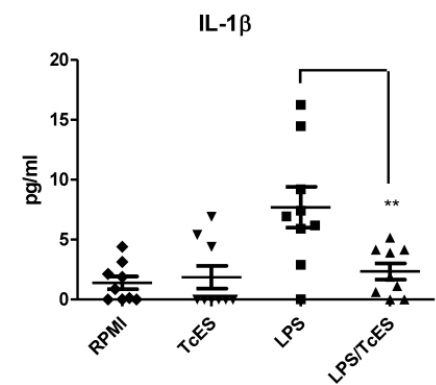

IL-6

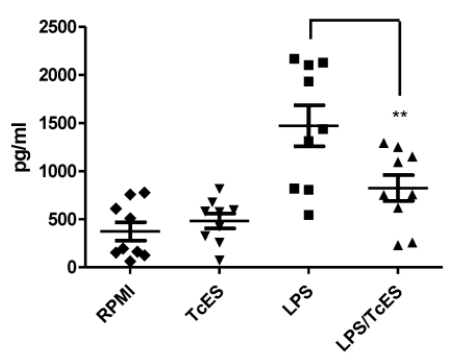

B)

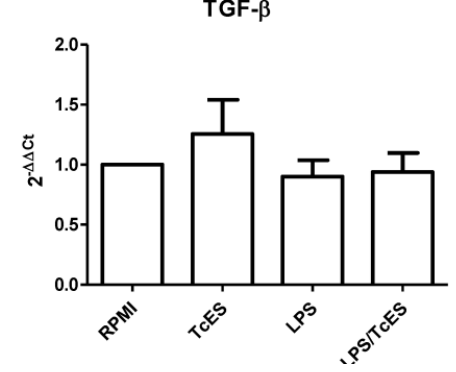

TNF

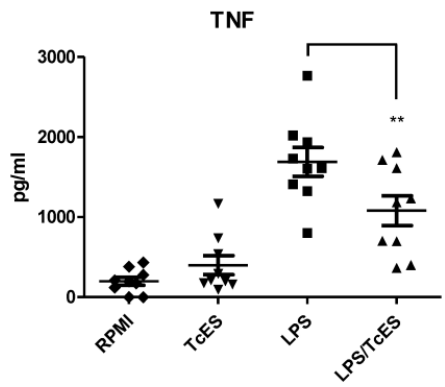

IL-10

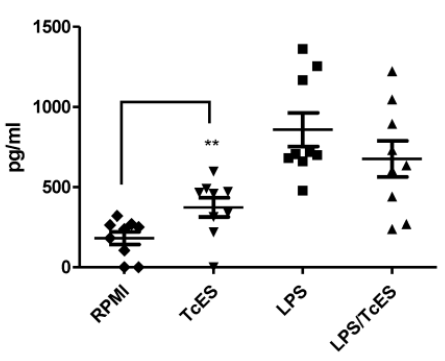

IL-23

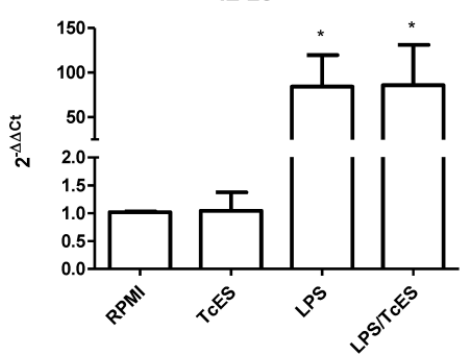

IL-12

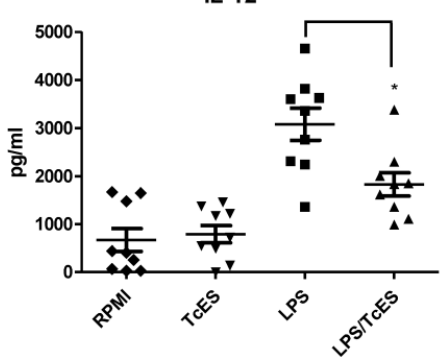

Figure 2. Cytokine response of human DCs exposed to TcES. Monocyte-derived DCs were stimulated with $20 \mu g$ of TcES, I $\mu \mathrm{g}$ of LPS or a combination of them. A) Supernatants were collected 24h post-stimulation and cytokine response was measured using commercial ELISA kits (Peprotech and R\&D). DCs derived from donors I-9 (nine donors) were used for this analysis. Assays were performed in duplicate. Data represents means \pm standard error of nine independent experiments. * P<0.05 was deemed significant. LPS vs RPMI was significantly different for all cytokines B) Cells were collected $3 \mathrm{~h}$ post-stimulation and RNA was extracted with TRIzol reagent and analysis of RT-qPCR was performed according to M\&M. DCs derived from donors 10-15 (six donors) were used for this analysis. Assays were performed in duplicate. Data represents means \pm standard error of six independent experiments. $* P<0.05$ was deemed significant.

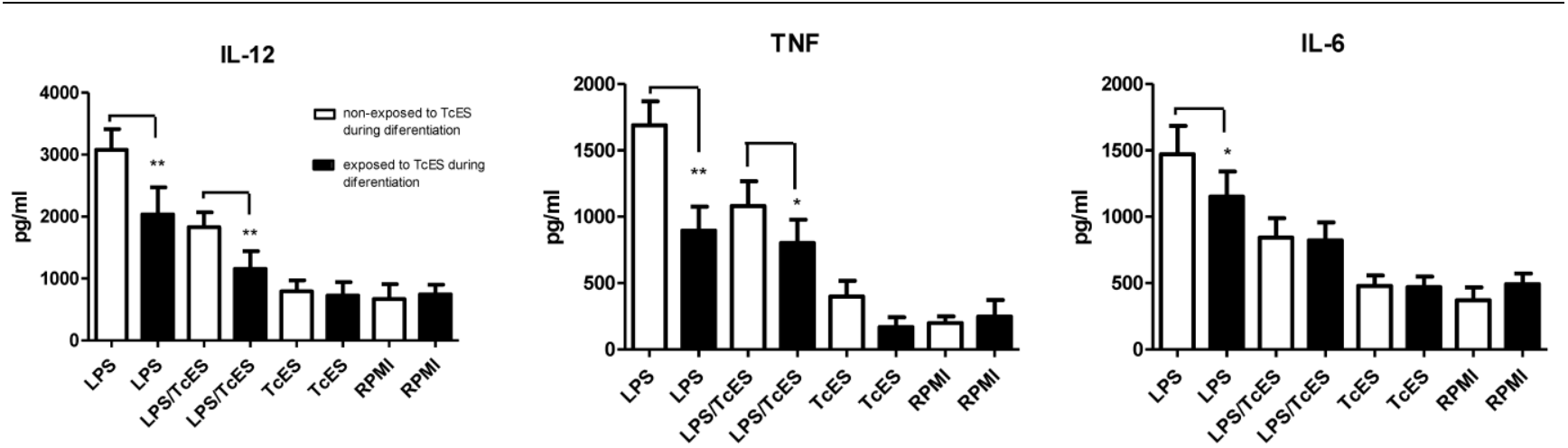

Figure 3. Comparison of cytokine response of human DCs exposed during differentiation from monocytes or at the end of their differentiation to TcES. Human monocytes were exposed to $20 \mathrm{ug} / \mathrm{ml}$ of TcES at day 0 and 3 of their differentiation to DCs. Once differentiated in iDCs they were stimulated with LPS, LPS+TcES or TcES alone. Supernatants were collected $24 \mathrm{~h}$ post-stimulation and production of cytokines measured using ELISA kits. DCs derived from donors I-9 (nine donors) were used for this analysis. Assays were performed in duplicate. Data represents means \pm standard error of nine independent experiments. $* \mathrm{P}<0.05$ was deemed significant. 

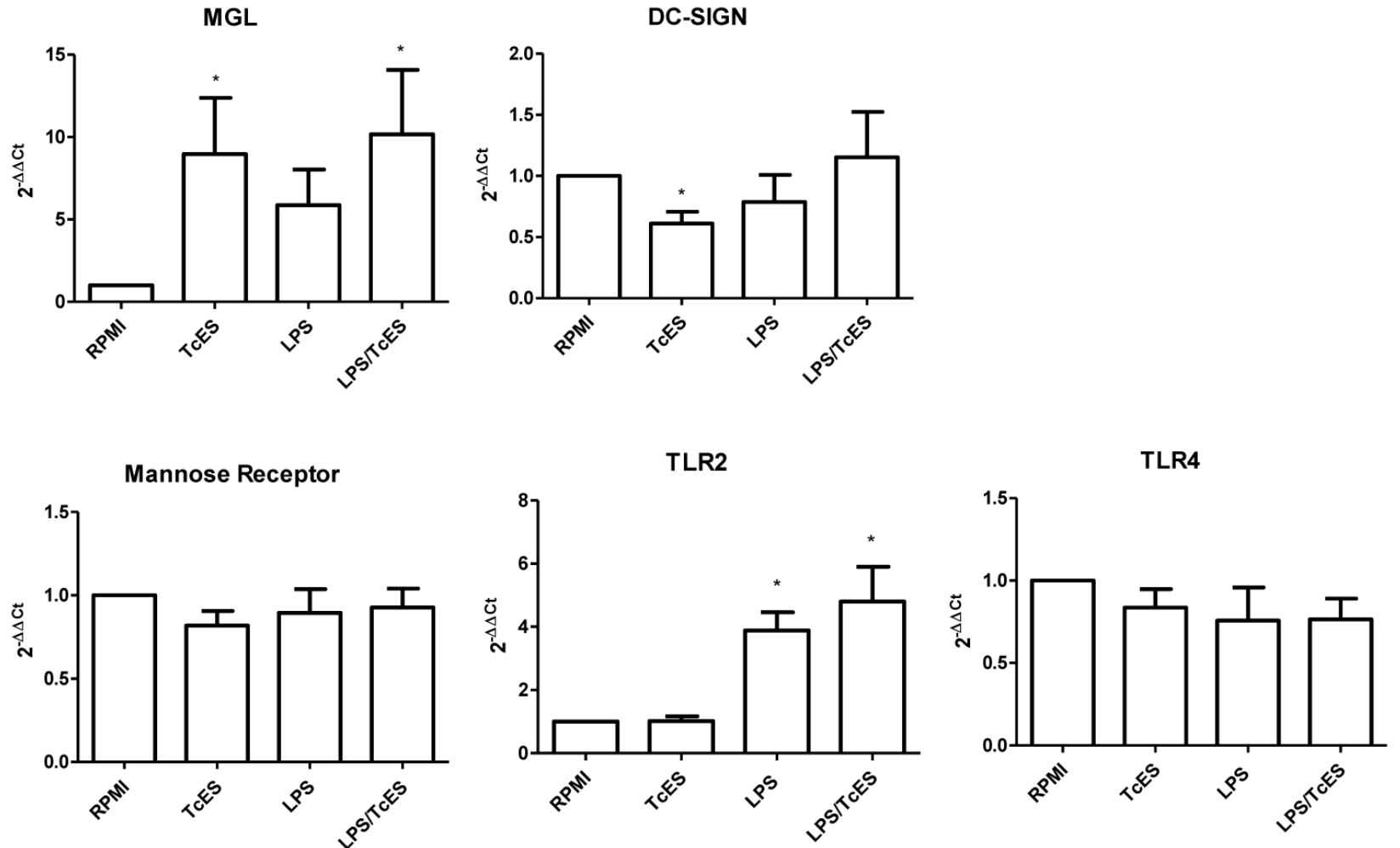

Figure 4. CLR and TLR expression in human DCs exposed to TcES. mRNA levels of MGL, DC-SIGN, MR, TLR4 and TLR2 were determined by RT-qPCR according to M\&M in human DC exposed during $3 \mathrm{~h}$ to $20 \mu \mathrm{g}$ of TcES, I $\mathrm{\mu g}$ of LPS or a combination of them. DCs derived from donors 10-15 (six donors) were used for analysis. Assays were performed in duplicate. Data represents means \pm standard error of six independent experiments. $* \mathrm{P}<0.05$ was deemed significant.

\section{TcES modulates expression of CLRs but not TLRs in human DCs}

In order to assess if changes induced in DC phenotype by TcES was extended to CLRs and TLRs previously reported as important in the responses to helminth antigens [25], we performed RT-qPCR analysis to human DCs exposed to TcES, LPS and the combination of them.

We found that TcES modulate positively the transcription of the C-type lectin receptor MGL while LPS failed to do so (Fig 4). When the stimulus was a combination of TcES and LPS the MGL up-regulation was again observed, indicating that TcES is able to induce its transcriptional enhancement even in the presence of a complete different stimulus such as LPS. On the contrary, the transcription of another C-type lectin receptor, DC-SIGN, was diminished only in presence of TcES but not of LPS or LPS plus TcES. The mRNA expression of the mannose receptor was not modified under any stimuli tested in this study. Similarly, when we explored the activity of TcES on the expression of the two TLRs involved in LPS recognition, TLR2 and TL4, we did not observe any modulation (Fig 4).

\section{Discussion}

In this work we demonstrated that the excreted-secreted antigens of the cestode T. crassiceps (TcES) modify the phenotype of human DCs. We found that when exposed to TcES, these cells acquired a phenotype compatible with tolerogenic DCs judged by their low levels of maturation markers such as CD83, CD80 and CD86 and the lack of secretion of inflammatory cytokines but instead an enhanced production of IL-10. The effects of TcES upon DCs were not limited to their capacity to induce a tolerogenic-like phenotype, since these antigens also down-modulated the pro-maturation and pro-inflammatory activities that LPS exerts on DCs, by reducing the expression of CD83, CD80, CD86 and the production of IL-12, TNF, IL-1 $\beta$ and IL- 6 but interestingly, not the one of IL-10.

Our results are in accordance with other reports describing that DCs show an "immature" phenotype after exposure to helminth antigens and where such exposure can also impair the subsequent TLR-mediated response induced in these cells [30,41]. However, IL-10 production by DCs exposed to helminth antigens has not been a common observation. 
For instance, others have found that DCs exposed to Echinococcus granulosus or Trichuris muris-derived antigens had an enhanced production of IL-10, however these cells also produced significant amounts of TNF and/or IL-6 [36, 42]. The induction of IL-10 by TcES may also possibly explain the apparent selective down-modulation of pro-inflammatory cytokines by TcES in DCs challenged with LPS. To our knowledge, this is the first study to report an enhanced production of the regulatory IL-10 by human DCs in response to helminth antigens, with no concomitant secretion of pro-inflammatory cytokines, reinforcing the idea of the induction of a tolerogenic-like phenotype in these cells by TcES. It will be crucial to address if these DCs, besides its tolerogenic phenotype, are consequently able of inducing $\mathrm{T}$ regulatory responses.

As we stated earlier, most of the tolerogenic DCs described to date display an immature like-phenotype characterized by a lower expression of co-stimulatory molecules, a decreased production of pro-inflammatory key cytokines such as IL-12 and a preferential production of regulatory cytokines such as IL-10 and TGF- $\beta$ [9-11]. Treatment of DCs with agents as IL-10 or corticoids stimulates their differentiation into a tolerogenic type [2, 7-8, 43]. Particularly, tolerogenic DCs that receive dexamethasone show a differential expression of the CLRs DC-SIGN, MR and MGL, where only the last one is up-regulated [14]. Interestingly, we found that human DCs exposed to TcES express a MGL transcriptional up-regulation, suggesting once again an induction of a tolerogenic phenotype in these cells. DC-SIGN is another CLR with important functions in DCs. This receptor not only supports recognition and internalization of pathogens for a later antigen presentation but it is also involved in shaping dendritic and $\mathrm{T}$ cell responses [44]. Interestingly, we observed that TcES but not LPS, down-modulated transcriptional expression of DC-SIGN in human DCs. In accordance with our findings, it has been shown that the human herpesvirus 6 [45], Leishmania donovani, L. major [46], and the helminth Brugia malayi [47] are able to reduce the expression of this CLR in DCs, presumably interfering with their function. Considering that the balance between the TLR and the CLR repertoire and their functional cooperation participate in the fine-tuning of regulatory mechanisms to allow appropriate immune responses, the over-expression of MGL in DCs exposed to TcES and the simultaneous down-regulation of DC-SIGN, may be part of the possible mechanisms used by $T$. crassiceps to modify the phenotype and hence the function of human DCs, directing the balance towards immune suppression and allowing in this way its own survival. We have previously shown that $T$. crassiceps antigens are heavily glycosilated [18] and that the carbohydrates present in these antigens are critical for their modulatory activities [18, 35, 48]. For these reasons, it is not surprising that TcES could modulate the expression of receptors such as MGL and DC-SIGN, which recognize sugar moieties. In fact, T. crassiceps antigens are glycosilated [49] with the specific carbohydrates known to be recognized at least by DC-SIGN. More studies are necessary to address if the mRNA pattern of expression of CLRs observed in this study in DCs exposed to TcES is also observed at the protein level, but yet more important is to determine if these receptors are involved in the recognition of and in the modulatory activity that $T$. crassiceps-antigens exert on human DCs.

Our results indicate that DCs challenged with TcES can impair their response to LPS by inhibiting the maturation process and reducing the secretion of pro-inflammatory cytokines such as IL-12, IL-1 $\beta$, TNF and IL-6, suggesting that TcES render these DCs unable to mature and probably incapable to support an inflammatory response after challenged with to a TLR stimulus. In this study we also examined the response of monocytes to TcES during their differentiation into DCs. In contrast with other studies that have shown that helminths or their products can affect the differentiation of monocytes into DCs [36-37], we found that exposure of monocytes to TcES did not affect the final percentage of $\mathrm{CD} 11 \mathrm{c}+$ cells, suggesting that differentiation of monocytes into DCs is not affected by TcES. However, when stimulated with LPS, TcES-differentiated DCs showed a significant reduction in the percentage of CD83+ cells and a more pronounced dropping in their production of IL-1 $\beta$, TNF and IL-6 when compared with DCs that were not exposed to TcES during their differentiation, suggesting that if monocytes encounter excreted/secreted antigens of $T$. crassiceps in their differentiation process to DCs, this could greatly affect their ability to mature and to respond to subsequent TLR stimulation. This is a plausible scenario to happen also in vivo since it has been reported that infections with metacestodes are accompanied with the release of antigens to circulation [38-39].

There has been a lot of speculation regarding the use of helminth antigens as modulators in autoimmune disorders [50-51]. Recent findings indicate that the cestode $T$. crassiceps is capable of ameliorating autoimmune pathologies in animal models [52]. Importantly, despite this cestode is not a human parasite in this work we demonstrate that its molecules have a modulatory activity upon human cells, opening the possibility to use these antigens as modulators in human disorders. A possible explanation for the con- 
served activity of TcES across species may simply rely on the immunogenic resemblance between the human cestode parasite T. solium and T. crassiceps $[32,53]$.

In summary, here we show that TcES induce a tolerogenic-like phenotype in human DCs as judged by the lack of enhancement of maturation and co-stimulatory molecules, an elevated production of IL-10 and the up-regulation of MGL expression. Additionally, these antigens are capable of impair the LPS pro-inflammatory activity in the same cells, down-modulating their maturation and secretion of inflammatory cytokines.

\section{Acknowledgments}

This work was supported by Instituto Nacional de CardiologÍa Ignacio Chávez. To laboratory technician Angeles Soster Contreras for her assistance to obtain blood samples.

\section{Conflict of Interests}

The authors have declared that no conflict of interest exists.

\section{References}

1. Banchereau J, et al. Immunobiology of dendritic cells. Annu Rev Immunol, 2000. 18: 767-811.

2. Steinman R.M, Hawiger D, and Nussenzweig M.C. Tolerogenic dendritic cells. Annu Rev Immunol, 2003. 21: 685-711.

3. Goubier A, et al. Plasmacytoid dendritic cells mediate oral tolerance. Immunity, 2008. 29(3): 464-75.

4. Pulendran B, et al. Modulating the immune response with dendritic cells and their growth factors. Trends Immunol, 2001. 22(1): 41-7.

5. Jonuleit $\mathrm{H}$, et al. Induction of interleukin 10-producing, nonproliferating CD4(+) $\mathrm{T}$ cells with regulatory properties by repetitive stimulation with allogeneic immature human dendritic cells. J Exp Med, 2000. 192(9): 1213-22.

6. Dhodapkar M.V, et al. Antigen-specific inhibition of effector T cell function in humans after injection of immature dendritic cells. J Exp Med, 2001. 193(2): 233-8.

7. Piemonti L, et al. Glucocorticoids affect human dendritic cell differentiation and maturation. Immunol J, 1999. 162(11): 6473-81.

8. Steinbrink $\mathrm{K}$, et al. Induction of tolerance by IL-10-treated dendritic cells. Immunol J, 1997. 159(10): 4772-80.

9. Coombes J.L, et al. A functionally specialized population of mucosal CD103+ DCs induces Foxp3+ regulatory T cells via a TGF-beta and retinoic acid-dependent mechanism. J Exp Med, 2007. 204(8): 1757-64.

10. Akbari O, DeKruyff R.H, and Umetsu D.T. Pulmonary dendritic cells producing IL-10 mediate tolerance induced by respiratory exposure to antigen. Nat Immunol, 2001. 2(8): 725-31.

11. Roncarolo M.G, Levings M.K, and Traversari C. Differentiation of $\mathrm{T}$ regulatory cells by immature dendritic cells. J Exp Med, 2001. 193(2): F5-9.

12. Ghiringhelli $\mathrm{F}$, et al. Tumor cells convert immature myeloid dendritic cells into TGF-beta-secreting cells inducing CD4+CD25+ regulatory T cell proliferation. J Exp Med, 2005. 202(7): 919-29.

13. Geijtenbeek T.B, et al. Self- and nonself-recognition by C-type lectins on dendritic cells. Annu Rev Immunol, 2004. 22: 33-54.
14. van Vliet S.J, et al. Differential regulation of C-type lectin expression on tolerogenic dendritic cell subsets. Immunobiology, 2006. 211(6-8): 577-85.

15. Appelmelk B.J, et al. Cutting edge: carbohydrate profiling identifies new pathogens that interact with dendritic cell-specific ICAM-3-grabbing nonintegrin on dendritic cells. Immunol J, 2003. 170(4): 1635-9.

16. Geijtenbeek T.B, et al. Mycobacteria target DC-SIGN to suppress dendritic cell function. J Exp Med, 2003. 197(1): 7-17.

17. Gantner B.N, et al. Collaborative induction of inflammatory responses by dectin-1 and Toll-like receptor 2. J Exp Med, 2003. 197(9): 1107-17.

18. Gomez-Garcia L, et al. Intact glycans from cestode antigens are involved in innate activation of myeloid suppressor cells. Parasite Immunol, 2005. 27(10-11): 395-405.

19. Geijtenbeek T.B and Gringhuis S.I. Signalling through C-type lectin receptors: shaping immune responses. Nat Rev Immunol, 2009. 9(7): 465-79.

20. Maizels R.M, et al. Regulation of pathogenesis and immunity in helminth infections. J Exp Med, 2009. 206(10): 2059-66.

21. Semnani R.T, et al. Filaria-induced immune evasion: suppression by the infective stage of Brugia malayi at the earliest host-parasite interface. Immunol J, 2004. 172(10): 6229-38.

22. Sciutto E, et al. Depressed T-cell proliferation associated with susceptibility to experimental Taenia crassiceps infection. Infect Immun, 1995. 63(6): 2277-81.

23. Reyes J.L and Terrazas L.I. The divergent roles of alternatively activated macrophages in helminthic infections. Parasite Immunol, 2007. 29(12): 609-19.

24. Segura $M$, et al. Impairment of dendritic cell function by excretory-secretory products: a potential mechanism for nematode-induced immunosuppression. Eur Immunol J, 2007. 37(7): 1887-904.

25. Terrazas C.A, Terrazas L.I, and Gomez-Garcia L. Modulation of dendritic cell responses by parasites: a common strategy to survive. J Biomed Biotechnol. 2010;: 357106.

26. Kean D.E, et al. Dissecting Ascaris glycosphingolipids for immunomodulatory moieties--the use of synthetic structural glycosphingolipid analogues. Parasite Immunol, 2006. 28(3): 69-76.

27. Jenkins S.J and Mountford A.P. Dendritic cells activated with products released by schistosome larvae drive Th2-type immune responses, which can be inhibited by manipulation of CD40 costimulation. Infect Immun, 2005. 73(1): 395-402.

28. Balic A, et al. Selective maturation of dendritic cells by Nippostrongylus brasiliensis-secreted proteins drives Th2 immune responses. Eur Immunol J, 2004. 34(11): 3047-59.

29. Dowling D.J, et al. Major secretory antigens of the helminth Fasciola hepatica activate a suppressive dendritic cell phenotype that attenuates Th17 cells but fails to activate Th2 immune responses. Infect Immun. 2010;78(2): 793-801.

30. van Liempt $\mathrm{E}$, et al. Schistosoma mansoni soluble egg antigens are internalized by human dendritic cells through multiple C-type lectins and suppress TLR-induced dendritic cell activation. Mol Immunol, 2007. 44(10): 2605-15.

31. Espindola N.M, et al. Cysticercosis immunodiagnosis using 18and 14-kilodalton proteins from Taenia crassiceps cysticercus antigens obtained by immunoaffinity chromatography. J Clin Microbiol, 2005. 43(7): 3178-84.

32. Toledo A, et al. Two epitopes shared by Taenia crassiceps and Taenia solium confer protection against murine T. crassiceps cysticercosis along with a prominent $\mathrm{T} 1$ response. Infect Immun, 2001. 69(3): 1766-73.

33. Villa O.F and Kuhn R.E. Mice infected with the larvae of Taenia crassiceps exhibit a Th2-like immune response with concomitant anergy and downregulation of Th1-associated phenomena. Parasitology, 1996. 112 ( Pt 6): 561-70. 
34. Rodriguez-Sosa M, et al. A STAT4-dependent Th1 response is required for resistance to the helminth parasite Taenia crassiceps. Infect Immun, 2004. 72(8): 4552-60.

35. Terrazas C.A, Gomez-Garcia L, and Terrazas L.I. Impaired pro-inflammatory cytokine production and increased Th2-biasing ability of dendritic cells exposed to Taenia excreted/secreted antigens: A critical role for carbohydrates but not for STAT6 signaling. Int J Parasitol. 2010;40(9): 1051-62.

36. Rigano R, et al. Echinococcus granulosus antigen B impairs human dendritic cell differentiation and polarizes immature dendritic cell maturation towards a Th2 cell response. Infect Immun, 2007. 75(4): 1667-78.

37. Fujiwara R.T, et al. Necator americanus infection: a possible cause of altered dendritic cell differentiation and eosinophil profile in chronically infected individuals. PLoS Negl Trop Dis, 2009. 3(3): e399.

38. Nguekam A, et al. Kinetics of circulating antigens in pigs experimentally infected with Taenia solium eggs. Vet Parasitol, 2003. 111(4): 323-32.

39. Alexander A.M, et al. Long-term clinical evaluation of asymptomatic subjects positive for circulating Taenia solium antigens. Trans R Soc Trop Med Hyg. 2010;104(12): 809-10.

40. Cronin S.J and Penninger J.M. From T-cell activation signals to signaling control of anti-cancer immunity. Immunol Rev, 2007. 220: $151-68$

41. Hamilton C.M, et al. The Fasciola hepatica tegumental antigen suppresses dendritic cell maturation and function. Infect Immun, 2009. 77(6): 2488-98.

42. D'Elia R and Else K.J. In vitro antigen presenting cell-derived IL-10 and IL-6 correlate with Trichuris muris isolate-specific survival. Parasite Immunol, 2009. 31(3): 123-31.

43. Moore K.W, et al. Interleukin-10 and the interleukin-10 receptor. Annu Rev Immunol, 2001. 19: 683-765.

44. Svajger U, et al. C-type lectin DC-SIGN: an adhesion, signalling and antigen-uptake molecule that guides dendritic cells in immunity. Cell Signal. 2010;22(10): 1397-405.

45. Niiya $\mathrm{H}$, et al. Transcriptional downregulation of DC-SIGN in human herpesvirus 6-infected dendritic cells. J Gen Virol, 2004. 85(Pt 9): 2639-42.

46. Revest $\mathrm{M}$, et al. Comparison of the immunomodulatory effects of donovani L and L. major excreted-secreted antigens, particulate and soluble extracts and viable parasites on human dendritic cells. Vaccine, 2008. 26(48): 6119-23.

47. Talaat K.R, et al. Preexposure to live Brugia malayi microfilariae alters the innate response of human dendritic cells to Mycobacterium tuberculosis. J Infect Dis, 2006. 193(2): 196-204.

48. Gomez-Garcia L, et al. Carbohydrate components of Taenia crassiceps metacestodes display Th2-adjuvant and anti-inflammatory properties when co-injected with bystander antigen. Parasitol Res, 2006. 99(4): 440-8.

49. Lee J.J, et al. Mass spectrometric characterisation of Taenia crassiceps metacestode N-glycans. Mol Biochem Parasitol, 2005. 143(2): 245-9.

50. McKay D.M. The therapeutic helminth? Trends Parasitol, 2009. 25(3): 109-14.

51. Wilson M.S and Maizels R.M. Regulation of allergy and autoimmunity in helminth infection. Clin Rev Allergy Immunol, 2004. 26(1): 35-50.

52. Argueta-Donohue J, et al. Leishmania mexicana: participation of NF-kappaB in the differential production of IL-12 in dendritic cells and monocytes induced by lipophosphoglycan (LPG). Exp Parasitol, 2008. 120(1): 1-9.

53. Peralta R.H, et al. Taenia crassiceps cysticerci: Characterization of the 14-kDa glycoprotein with homologies to antigens from Taenia solium cysticerci. Exp Parasitol, 2010 Mar;124(3):295-300. 